\title{
CORRECTION
}

\section{Correction: Association between the National Cancer Screening Programme (NSCP) for gastric cancer and oesophageal cancer mortality}

Jie-Hyun Kim, Kyung-Do Han, Jung Kuk Lee, Hyun-Soo Kim, Jae Myung Cha, Sohee Park, Joo Sung Kim, Won Ho Kim and for the Big Data Research Group (BDRG) of the Korean Society of Gastroenterology (KSG)

British Journal of Cancer (2020) 123:496; https://doi.org/10.1038/s41416-020-0946-z

Correction to: British Journal of Cancer (2020); https://doi.org/ 10.1038/s41416-020-0883-x, published online 13 May 2020

Since the publication of this paper, the authors have noticed an error in the name and department of author Sohee Park. Dr. Park's name was incorrectly listed as So Hee Park. The Department of Biostatistics, Graduate School of Public Health was incorrectly listed as the Department of Public Health, Graduate School. Both errors have been corrected above. The authors apologise for this oversight. 